\title{
Alantolactone and isoalantolactone suppress maximal electroshock-induced tonic seizures in mice
}

\author{
Jarogniew J. Łuszczki ${ }^{1,2}$, Ewa Marzęda', Maria W. Kondrat-Wróbel ${ }^{2}$, Magdalena Florek-Łuszczki \\ ${ }^{1}$ Isobolographic Analysis Laboratory, Institute of Rural Health in Lublin, Poland \\ 2 Department of Pathophysiology, Medical University of Lublin in Lublin, Poland \\ ${ }^{3}$ Department of Public Health, Institute of Rural Health in Lublin, Poland
}

Łuszczki JJ, Marzęda E, Kondrat-Wróbel MW, Florek-Łuszczki M. Alantolactone and isoalantolactone suppress maximal electroshock-induced tonic seizures in mice. J Pre-Clin Clin Res. 2014; 8(1): 9-12.

\section{Abstract}

Introduction and objective. The aim of this study was to perform the anticonvulsant screening test to determine whether two sesquiterpene lactones (alantolactone and isoalantolactone) isolated from herbs and medicinal plants offer a distinct protection against maximal electroshock (MES)-induced tonic seizures in mice.

Materials and methods. The screening test was performed for alantolactone and isoalantolactone administered intraperitoneally in a constant dose of $300 \mathrm{mg} / \mathrm{kg}$ at 4 various pretreatment times (i.e., 15, 30, 60 and $120 \mathrm{~min}$.) before the MES test. Tonic hind limb extension (seizure activity) was evoked in adult male albino Swiss mice by a current (sine-wave, $25 \mathrm{~mA}, 500 \mathrm{~V}, 50 \mathrm{~Hz}, 0.2 \mathrm{~s}$ stimulus duration) delivered via ear-clip electrodes. Subsequently, the median effective doses $\left(\mathrm{ED}_{50}\right.$ values) for alantolactone and isoalantolactone were determined in the MES test in mice.

Results. In the screening test, both compounds produced a $37.5 \%$ protection against MES-induced tonic seizures in mice, when administered i.p. at $15 \mathrm{~min}$. prior to the MES test. In contrast, alantolactone and isoalantolactone administered i.p. at 30, 60 and $120 \mathrm{~min}$. prior to the test produced no anticonvulsant activity in mice subjected to the MES test. The experimentallyderived $\mathrm{ED}_{50}$ values for alantolactone and isoalantolactone, administered intraperitoneally at $15 \mathrm{~min}$. before the MES test, were $322(281-369) \mathrm{mg} / \mathrm{kg}$ and $336(285-396) \mathrm{mg} / \mathrm{kg}$, respectively.

Conclusions. Alantolactone and isoalantolactone ( 2 sesquiterpene lactones) are worth considering as potentially favourable compounds in epileptology, if the results from this study could be extrapolated into clinical settings.

\section{Key words}

alantolactone, isoalantolactone, maximal electroshock seizure test

\section{INTRODUCTION}

No doubt exists that medicinal plants have a long history of use in the treatment of human diseases. There is still continued interest in the investigation of extracts of medicinal plants in the search for some novel bioactive molecules $[1,2,3,4,5,6]$. Indeed, bioactive molecules isolated from medicinal plants have played a principal role in the discovery of compounds for the development of drugs for the treatment of most human diseases, including epilepsy $[4,5,6]$. At present, researchers and clinicians are trying to find some novel antiepileptic drugs among various naturally occurring bioactive molecules isolated from herbs and medicinal plants that could inhibit seizure activity in patients with refractory epilepsy $[4,5,6]$. Our previous studies have documented that imperatorin, osthole and xanthotoxin (3 naturally occurring coumarins) produced anticonvulsant action in the mouse maximal electroshock (MES)-induced seizure model [7, 8, 9, 10, 11]. In contrast, 2 other coumarins, bergapten and oxypeucedanin had no impact on MES-induced tonic seizures in mice [9]. Additionally, it was found that borneol and thymoquinone produced the anticonvulsant action in the mouse MES model [12].

Address for correspondence: J.J. Luszczki, Institute of Rural Health, Jaczewskiego 2, 20-090 Lublin, Poland

e-mail: jluszczki@yahoo.com or jarogniew.luszczki@gmail.com

tel.: (+48) 817184488

Received: 10 January 2014; accepted: 16 May 2014
Sesquiterpene lactones (alantolactone and isoalantolactone (Fig. 1) isolated from Inula helenium L. (growing in Poland) have been reported to have various pharmacological activities, including anti-inflammatory $[13,14]$, antimicrobial $[15,16]$, antifungal, antihelmintic $[17,18]$ and antiproliferative $[19$, 20] properties. Additionally, isoalantolactone protects against Staphylococcus aureus pneumonia [21].

Considering the above-mentioned facts, we attempted to perform the first anticonvulsant screening test to determine whether or not these sesquiterpene lactones (alantolactone and isoalantolactone) possess the anticonvulsant properties in the mouse MES model. It is widely accepted that the mouse MES model allows selecting agents possessing the anticonvulsant activity, because in this test, several classical, second- and third-generation antiepileptic drugs (including, carbamazepine, phenytoin, phenobarbital, valproate, lamotrigine, oxcarbazepine, lacosamide, retigabine, pregabalin and topiramate), exert the anticonvulsant action by suppressing MES-induced tonic seizures in mice [22].

\section{MATERIALS AND METHOD}

Animals and experimental conditions. Adult male Swiss mice (weighing 22-26 g) were kept in colony cages with free access to food and tap water, under standardized housing conditions (natural light-dark cycle, temperature $23 \pm 1^{\circ} \mathrm{C}$, relative humidity $55 \pm 5 \%$ ), were used. After 7 days 
of adaptation to laboratory conditions, the animals were randomly assigned to experimental groups comprising of 8 mice in each group. Each mouse was used only once, and all tests were performed between 08.00-15.00 hours. Procedures involving animals and their care were conducted in accordance with current European Community and Polish legislation on animal experimentation. Additionally, all efforts were made to minimize animal suffering and to use only the number of animals necessary to produce reliable scientific data. The experimental protocols and procedures described in this study were approved by the Second Local Ethics Committee at the University of Life Sciences in Lublin (License No. 85/2009), and complied with the European Communities Council Directive of 24 November 1986 (86/609/EEC).

Substances. The following substances were used in this study: alantolactone [IUPAC name: (3aR,5S,8aR,9aR)-5,8adimethyl-3-methylidene-5, 6,7,8,9,9a-hexahydro-3aHbenzo[f][1]benzofuran-2-one (Fig. 1) and isoalantolactone [IUPAC name: (3aR,4aS,8aR,9aR)-8a-methyl-3,5dimethylidene-3a,4,4a,6,7,8,9,9a-octahydrobenzo[f][1] benzofuran-2-one (Fig. 1)]. Both compounds were purchased from ChromaDex (Irvine, CA, USA), and samples of $1 \mathrm{~g}$ for the screening test were provided by Dr. M. Gleńsk and Dr. M. Włodarczyk from the Department of Pharmacognosy at the Medical University in Wroclaw, Poland. All substances were suspended in a $1 \%$ solution of Tween 80 (Sigma, St. Louis, MO, USA) in distilled water and administered intraperitoneally (i.p.) as a single injection, in a volume of $5 \mathrm{ml} / \mathrm{kg}$ body wt. In the screening test, the studied compounds were administered i.p. at a constant dose of $300 \mathrm{mg} / \mathrm{kg}$ at 4 pretreatment times (i.e., 15, 30, 60 and $120 \mathrm{~min}$ ) before the MES test.

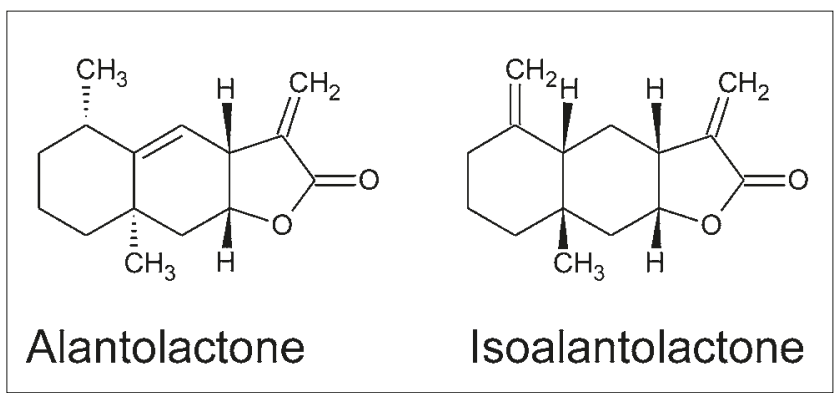

Figure 1. Structural formulas of alantolactone and isoalantolactone

Maximal electroshock-induced seizures. Electroconvulsions were produced by means of an alternating current (sine-wave, $0.2 \mathrm{~s}$ stimulus duration, $50 \mathrm{~Hz}, 25 \mathrm{~mA}, 500 \mathrm{~V}$ ) delivered via ear-clip electrodes by a Type 221Rodent Shocker generator (Hugo Sachs Elektronik, Freiburg, Germany). The electrical system of the stimulator was self-adjustable so that changes in impedance did not result in alterations of current intensity (i.e., the system provides constant current stimulation). The criterion for the occurrence of seizure activity was the tonic hind limb extension (i.e., the hind limbs of animals outstretched $180^{\circ}$ to the plane of the body axis). The protective activity of alantolactone and isoalantolactone in the screening test was determined as the percentage of protection of animals against MES-induced tonic seizures.

Additionally, the animals were administered with different increasing doses of alantolactone and isoalantolactone in order to obtain a variable percentage of protection against MES-induced seizures, allowing the construction of a doseresponse relationship curve for each compound administered alone. Each $\mathrm{ED}_{50}$ value represented the dose of alantolactone or isoalantolactone required to protect half of the animals tested against MES-induced tonic seizures. In the presented study, to determine the $\mathrm{ED}_{50}$ values of alantolactone and isoalantolactone, both compounds were administered i.p. at the dose ranges $300-400 \mathrm{mg} / \mathrm{kg}$. Using the log-probit method, the median effective doses $\left(\mathrm{ED}_{50}\right.$ values) were determined using a minimum of 8 mice per dose [23], after which the mice were euthanized by $\mathrm{CO}_{2}$ narcosis.

\section{RESULTS}

Influence of alantolactone and isoalantolactone on maximal electroshock-induced seizures in mice in the screening test. In the screening test, alantolactone and isoalantolactone administered i.p. at a constant dose of $300 \mathrm{mg} / \mathrm{kg}$ produced the anticonvulsant effect against MES-induced tonic seizures in mice at the respective pretreatment times (Tab. 1). Both compounds protected 3 out of 8 mice (37.5\% of animals tested) when administered i.p. at $15 \mathrm{~min}$. before the MES test (Tab. 1). Prolongation of the pretreatment times to 30, 60 and $120 \mathrm{~min}$. before the MES test produced no protection against tonic seizures in mice (Tab. 1). The experimentally-derived $\mathrm{ED}_{50}$ value for alantolactone administered i.p. at $15 \mathrm{~min}$. before the MES test was 322 (281-369) mg/kg. Simultaneously, the $\mathrm{ED}_{50}$ value of isoalantolactone administered i.p. at $15 \mathrm{~min}$. prior to the MES test was 336 (285-396) $\mathrm{mg} / \mathrm{kg}$.

Table 1. Time-course of anticonvulsant effects of alantolactone and isoalantolactone against maximal electroshock (MES)-induced seizures in mice

\begin{tabular}{|c|c|c|c|c|}
\hline Substance Time (min.) & $\begin{array}{c}15 \\
\mathrm{p} / \mathrm{t}(\%)\end{array}$ & $\begin{array}{c}30 \\
\mathrm{p} / \mathrm{t}(\%)\end{array}$ & $\begin{array}{c}60 \\
\mathrm{p} / \mathrm{t}(\%)\end{array}$ & $\begin{array}{c}120 \\
p / t(\%)\end{array}$ \\
\hline Alantolactone & $3 / 8(37.5)$ & $0 / 8(0)$ & $0 / 8(0)$ & $0 / 8(0)$ \\
\hline Isoalantolactone & $3 / 8(37.5)$ & $0 / 8(0)$ & $0 / 8(0)$ & $0 / 8(0)$ \\
\hline
\end{tabular}

Data are presented as number of animals protected against maximal electroshock (MES)-induced seizures out of 8 animals per group (and as \% in parentheses). The MES test was performed at various pretreatment times $(15,30,60$ and $120 \mathrm{~min}$.) after systemic (i.p.) administration of the investigated compounds in a fixed dose of $300 \mathrm{mg} / \mathrm{kg}$. p - number of animals protected against MES-induced seizures; $t$ - number of animals tested per group

\section{DISCUSSION}

The results obtained clearly indicate that alantolactone and isoalantolactone (2 sesquiterpene lactones) suppressed MES-induced tonic seizures in mice. It was found that both naturally occurring substances administered systemically (i.p.) at a constant dose of $300 \mathrm{mg} / \mathrm{kg}$ produced a moderate anticonvulsant action in the mouse MES test, because $37.5 \%$ of the animals tested were protected against MESinduced seizures. Evaluation of the time-course of the anticonvulsant effect for these compounds revealed that both compounds produced antielectroshock action when administered i.p. at $15 \mathrm{~min}$. prior to the test. Prolongation of the pretreatment times to 30,60 and $120 \mathrm{~min}$. before the electroconvulsions resulted in no anticonvulsant effect in the mouse MES model. It is worth mentioning that in the presented study the pretreatment times and doses of tested compounds were identical to those described previously in 
the screening test allowing selection of compounds with anticonvulsant activity $[9,11,12,24]$. Subsequently, the $\mathrm{ED}_{50}$ values of both compounds in the MES test were determined - 322 (281-369) $\mathrm{mg} / \mathrm{kg}$ for alantolactone and 336 (285-396) $\mathrm{mg} / \mathrm{kg}$ for isoalantolactone. Of note, the $\mathrm{ED}_{50}$ values for alantolactone and isoalantolactone in the mouse MES model are quite similar to those denoted for valproate [11]. It is widely accepted that valproate is the most active antiepileptic drug, which suppresses tonic, clonic, myoclonic, and absence of psychomotor seizures in experimental animals [25, 26]. Moreover, valproate has a wide range of therapeutic indications in epilepsy (for more details see [27]). Considering the above-mentioned facts, it is possible that alantolactone and isoalantolactone could also be effective in suppressing various seizure types. However, to confirm this hypothesis, more advanced neurochemical studies are required.

Previously, we documented that some naturally occurring compounds produced the anticonvulsant action in the mouse MES model. More specifically, it was found that borneol, thymoquinone [12], xanthotoxin [9], imperatorin and osthole $[7,8,11]$, produced a definite (clear-cut) anticonvulsant action in the mouse MES model. In contrast, several naturally occurring substances, including alizarin, arbutin, betulin, curcumin, diosmin, esculetin, ellagic acid, gallic acid, hesperidine, linalool, menthofuran, piperitol, piperonal, quercetin, alpha-terpineol, theobromine, beta-thujaplicin, ursolic acid and vanillin, produced no or negligible anticonvulsant activity in mice challenged with the screening procedure in the mouse MES model $[12,24]$.

From the results obtained, it can be concluded that structural modification of alantolactone and isoalantolactone may contribute to the increase in the antielectroshock activity of these 2 compounds. Perhaps chemical modification of their molecules may result in the discovery of new, potentially favourable drugs that could be used in patients with epilepsy.

Another potentially important finding of the presented study is that alantolactone and isoalantolactone possess anticonvulsant action which occurs at $15 \mathrm{~min}$. after their systemic administration. Generally, a fast anticonvulsant activity of drugs or active compounds is useful to stop seizure attacks in epileptic patients. It is possible to create a pharmaceutical formula of alantolactone and/or isoalantolactone that could be administered intravenously. In such a situation, a bolus of alantolactone or isoalantolactone could be given to the patients to stop their seizure attacks. However, more advanced neurochemical studies are required to elucidate the exact molecular mechanisms of the anticonvulsant action of both sesquiterpene lactones.

It is worth mentioning that the screening procedure applied in this study was almost identical to that accepted by National Institutes of Health (USA) and Antiepileptic Drug Development Programme for searching for novel antiepileptic drugs $[25,26]$. There is no doubt that bioactive compounds isolated from herbs and medicinal plants are a very important source in the search for compounds possessing anticonvulsant properties in preclinical studies. Herbs and plants usually contain a series of compounds that could be clinically favourable, especially, in epileptic patients.

Additionally, the tested compounds administered systemically (i.p.) at a constant dose of $300 \mathrm{mg} / \mathrm{kg}$ produced no adverse effects in mice. In other words, no signs of ataxia or motor coordination impairment were observed in this study in animals receiving alantolactone and isoalantolactone (results not shown).

Summing up, alantolactone and isoalantolactone (two sesquiterpene lactones) could be used in patients with epilepsy, if the results from this study would be extrapolated into clinical settings.

\section{Acknowledgements}

The study was supported by a grant from the Institute of Rural Health inLublin, Poland. The authors express their thanks to Dr. M. Gleńsk and Dr. M. Włodarczyk from the Department of Pharmacognosy at the Medical University in Wroclaw, Poland, for kindly providing samples of alantolactone and isoalantolactone for the experiments. Professor J. J. Luszczki is a Member of the Academy of Young Scholars, Polish Academy of Sciences in Warsaw, Poland.

\section{Disclosure of conflicts of interest}

The authors have no disclosures to declare.

\section{REFERENCES}

1. Mishra BB, Tiwari VK. Natural products: an evolving role in future drug discovery. Eur J Med Chem. 2011; 46: 4769-4807.

2. Koehn FE, Carter GT. The evolving role of natural products in drug discovery. Nat Rev Drug Discov. 2005; 4: 206-220.

3. Koehn FE, Carter GT. Rediscovering natural products as a source of new drugs. Discov Med. 2005; 5: 159-164

4. Christen P, Cuendet M. Plants as a source of therapeutic and health products. Chimia (Aarau). 2012; 66: 320-323.

5.Zhu HL, Wan JB, Wang YT, Li BC, Xiang C, He J, Li P. Medicinal compounds with antiepileptic/anticonvulsant activities. Epilepsia 2013; doi:10.1111/epi.12463. [in press].

6. Tagarelli G, Tagarelli A, Liguori M, Piro A. Treating epilepsy in Italy between XIX and XX century. J Ethnopharmacol. 2013; 145: 608-613.

7.Łuszczki JJ, Głowniak K, Czuczwar SJ. Imperatorin enhances the protective activity of conventional antiepileptic drugs against maximal electroshock-induced seizures in mice. Eur J Pharmacol. 2007; 574: 133-139.

8. Łuszczki JJ, Głowniak K, Czuczwar SJ. Time-course and dose-response relationships of imperatorin in the mouse maximal electroshock seizure threshold model. Neurosci Res. 2007; 59: 18-22.

9. Łuszczki JJ, Andres-Mach M, Gleńsk M, Skalicka-Woźniak K. Anticonvulsant effects of four linear furanocoumarins, bergapten, imperatorin, oxypeucedanin, and xanthotoxin, in the mouse maximal electroshock-induced seizure model: a comparative study. Pharmacol Rep. 2010; 62: 1231-1236.

10.Łuszczki JJ, Wojda E, Raszewski G, Głowniak K, Czuczwar SJ. Influence of imperatorin on the anticonvulsant activity and acute adverse-effect profile of lamotrigine in maximal electroshock-induced seizures and chimney test in mice. Pharmacol Rep. 2008; 60: 566-573.

11.Łuszczki JJ, Wojda E, Andres-Mach M, Cisowski W, Gleńsk M, Głowniak K, Czuczwar SJ. Anticonvulsant and acute neurotoxic effects of imperatorin, osthole and valproate in the maximal electroshock seizure and chimney tests in mice: a comparative study. Epilepsy Res. 2009; 85: 293-299.

12. Łuszczki JJ, Włodarczyk M, Gleńsk M, Marzęda E, Durmowicz D, Florek-Łuszczki M. Effects of various naturally occurring compounds (arbutin, borneol, esculetin, esculin, ellagic acid, gallic acid, hesperidine, piperitol, piperonal, quercetin, thymoquinone and ursolic acid) against maximal electroshock-induced seizures in mice. Curr Issues Pharm Med Sci. 2013; 26: 135-139.

13. Chun J, Choi RJ, Khan S, Lee DS, Kim YC, Nam YJ, Lee DU, Kim YS. Alantolactone suppresses inducible nitric oxide synthase and cyclooxygenase-2 expression by down-regulating NF- $\kappa \mathrm{B}, \mathrm{MAPK}$ and AP-1 via the MyD88 signaling pathway in LPS-activated RAW 264.7 cells. Int Immunopharmacol. 2012; 14: 375-383.

14. Schmidt TJ, Brun R, Willuhn G, Khalid SA. Anti-trypanosomal activity of helenalin and some structurally related sesquiterpene lactones. Planta Med. 2002; 68: 750-751. 
15. Stojanović-Radić Z, Comić Lj, Radulović N, Blagojević P, Denić M, Miltojević A, Rajković J, Mihajilov-Krstev T. Antistaphylococcal activity of Inula helenium L. root essential oil: eudesmane sesquiterpene lactones induce cell membrane damage. Eur J Clin Microbiol Infect Dis. 2012; 31: 1015-1025.

16.Xin XL, Ma XC, Liu KX, Han J, Wang BR, Guo DA. Microbial transformation of alantolactone by Mucor polymorphosporus. J Asian Nat Prod Res. 2008; 10: 933-937.

17. Tan RX, Tang HQ, Hu J, Shuai B. Lignans and sesquiterpene lactones from Artemisia sieversiana and Inula racemosa. Phytochemistry 1998; 49: 157-161.

18. Tripathi YB, Tripathi P, Upadhyay BN. Assessment of the adrenergic beta-blocking activity of Inula racemosa. J Ethnopharmacol. 1988; 23: 3-9.

19. Konishi T, Shimada Y, Nagao T, Okabe H, Konoshima T. Antiproliferative sesquiterpene lactones from the roots of Inula helenium. Biol Pharm Bull. 2002; 25: 1370-1372.

20. Pal HC, Sehar I, Bhushan S, Gupta BD, Saxena AK. Activation of caspases and poly (ADP-ribose) polymerase cleavage to induce apoptosis in leukemia HL-60 cells by Inula racemosa. Toxicol In Vitro. 2010; 24: 1599-1609.

21. Qiu J, Luo M, Wang J, Dong J, Li H, Leng B, Zhang Q, Dai X, Zhang Y, Niu X, Deng X. Isoalantolactone protects against Staphylococcus aureus pneumonia. FEMS Microbiol Lett. 2011; 324: 147-155.
22. Löscher W, Fassbender CP, Nolting B. The role of technical, biological and pharmacological factors in the laboratory evaluation of anticonvulsant drugs. II. Maximal electroshock seizure models. Epilepsy Res. 1991; 8: 79-94.

23. Litchfield JT, Wilcoxon F. A simplified method of evaluating dose-effect experiments. J Pharmacol Exp Ther. 1949; 96: 99-113.

24. Łuszczki JJ, Włodarczyk M, Gleńsk M, Marzęda E, Durmowicz D, Florek-Łuszczki M. Effects of alizarin, betulin, curcumin, diosmin, linalool, menthofuran, $\alpha$-terpineol, theobromine, $\beta$-thujaplicin and vanillin against maximal electroshock-induced seizures in mice. J PreClin Clin Res. 2013; 7: 5-8.

25. Stables JP, Kupferberg HJ. Chapter 16 - The NIH Anticonvulsant Drug Development (ADD) Program: preclinical anticonvulsant screening project. In: Avanzini G, Regesta G, Tanganelli P, Avoli M, eds. Molecular and cellular targets for anti-epileptic drugs. London, John Libbey, 1997. p. 191-198.

26. White HS, Woodhead JH, Wilcox KS, Stables JP, Kupferberg HJ, Wolf $\mathrm{HH}$. Discovery and preclinical development of antiepileptic drugs. In: Levy RH, Mattson RH, Meldrum BS, Perucca E, eds. Antiepileptic drugs. 5th ed. Philadelphia, Lippincott Williams \& Wilkins, 2002. p. 36-48.

27. Löscher W. Basic pharmacology of valproate: a review after 35 years of clinical use for the treatment of epilepsy. CNS Drugs 2002; 16: 669-694. 